\title{
The Turkish version of the İkigai-9 Scale: A validity and reliability study
}

Tahir Belice ( $\sim$ drtahirelf@hotmail.com )

SBU İzmir Bozyaka Training and Research Hospital Internal Diseases Department https://orcid.org/0000-0001-7957-3423

\section{Research Article}

Keywords: Depression, Turkey, Anxiety, İkigai, Well-being

Posted Date: February 2nd, 2022

DOI: https://doi.org/10.21203/rs.3.rs-1303957/v2

License: (c) (1) This work is licensed under a Creative Commons Attribution 4.0 International License. Read Full License 


\section{Abstract}

The aim: Ikigai, or well-being, has been associated with many positive outcomes in the physical and mental health of elderly people. In the present study, we conducted a validity and reliability study of the Ikigai-9 scale in Turkish society and outlined its associations with facets of well-being.

Methods: A translation, re-translation method was used to create linguistic equivalence of the İkigai-9 scale in the Turkish language. The correlations between the İkigai-9 Turkish version with the Warwick Edinburgh Mental Well-being Scale Short Form and the Depression Anxiety Stress Scale (DASS-21) were studied. Face-to-face interviews were conducted with volunteers regarding the scale. Data were saved in excel files and analyzed with SPSS (IBM SPSS for Windows, ver.24).

Results: The findings confirmed the validity and reliability of the İkigai-9 Turkish version using 382 participants. The total scores on the İigai-9 Turkish version were correlated positively with mental well-being and negatively correlated with depression, anxiety, and depression ( $<<0.05)$. Moreover, the total İkigai-9 Turkish version scores had a positive correlation with age $(p<0.05)$ but did not differ according to gender $(p>0.05)$.

Conclusions: İkigai and studies related to the concept of mental well-being should be widened and expanded across nations. These studies could be beneficial to healthcare providers to attenuate the impact of suffering from various diseases. We present the İkigai-9 Turkish version for further study in both in the national and international contexts that healthcare workers could compare their results in other countries.

\section{Introduction}

The concept of "ikigai" has remained popular as a comprehensive concept. Ikigai mainly describes subjective "well-being" in Japanese culture [1]. This concept reflects several meanings, including "enjoying life," "self-confidence," "being moral and ethical," and "happiness." The concept of ikigai also allows us to evaluate the meaning of life as reflected by the individual $[2,3,4]$. Ikigai appears to have a positive correlation with health status, based on several studies; therefore, an increase in studies on İkigai and improved arrangements to increase individual's levels of íkigai could lead to positive health-related and community outcomes [4,5].

The phrase "purpose of life" originates in 19th century European existential philosophy. İkigai, as a term, traces back to 14th century Japan [6,7]. Both purpose in life and İkigai are psychologically related to purpose and ambition in life. In the brain, this is located in the prefrontal lobe, which is also physiologically related to functions such as ambition, emotional regulation, and integration of psychological events [8,9]. Some psychiatric and somatic diseases, including depression and cardiovascular disease, could be exacerbated by anxiety that might evolve from a basis of life stress. Ensuring a state of subjective well-being has psychological and physical health benefits

The İkigai-9 was first developed by Imai et al. (2012) as psychometric scale in Chinese [10]. Fido et al. (2019) studied the Ikigai-9 for validation and reliability in the UK as an English version [10,11]. The İkigai-9 scale evaluates the individual's awareness of the way they derive purpose in life, the presence of active, positive attitudes and behaviors toward the future, and optimistism and positive emotions in their outlook on life [5]. With email permission from Dr. Fido, we conducted a validity and reliability study of the Turkish translation version of the ikigai-9. Our study aimed to demonstrate the reliability and validity of the ikigai-9 Turkish version with reference to the Warwick Edinburgh Mental Well-Being Scale Short Form and the Depression Anxiety Stress Scales (DASS-21) $[12,13]$. These scales have previously been proven as valid and reliable in Turkish.

\section{Objective}

In the present study, we confirmed the validity and reliability of the Ikigai- 9 Turkish version and propose for it to be made us of in further international studies as a tool to measure purpose in life.

\section{Methods}

\section{Data Collection and Study Design}

A total of 381 participants (male: 191, female: 191) were included in this study. G*Power ver. 3.1.9.4 was used to calculate the sample size with a 5\% type I error risk and $85 \%$ test power [14]. Inclusion criteria for this study were being over 18 years old and having the ability to answer and understand all the items in scale. Exclusion criteria were being under 18 years old and not able to to answer and understand all the items in scale. The study was performed using face-to-face interviews with the participants who were volunteers and provided informed consent (from November to 31 December 2019).

\section{Statistical Analysis}

Kolmogorov-Smirnov ( $\mathrm{n}>50)$ and Skewness-Kurtosis tests were used to check whether the measurements in the study were normally distributed and Parametric tests were applied since the measurements were normally distributed. Cronbach's Alpha coefficients were calculated within the scope of reliability analysis. Within the scope of Validity-

Reliability, "Factor Analysis" was applied to determine the "Ikigai Scale" sub-dimensions. We administered three scales and a demographic form. All the data was saved in excel documents. The means, percentages, medians and range were calculated in SPSS (IBM SPSS for Windows, ver.24). An independent ttest and one- 
way analysis of variance (ANOVA) were used to compare the variables. Linear Regression analysis was used to determine the effects of "depression, anxiety, stress, mental well-being and age" on "Ikigai" variable. Pearson

Correlation coefficients were calculated in determining the correlation. Statistical significance level (a) is taken as $5 \%$ in calculations.

\section{Permissions}

Prior to commencing, we requested permission via email/online to use the scales in our study, which were published by Dr. Demirtaş, Dr. Ozer and Dr. Fido. These included The Warwick Edinburgh Mental Well-being Scale, The Depression Anxiety Stress Scales (DASS) and The İkigai-9 UK scale.

\section{Ethics Approval}

Approval for this study was obtained from the ethics committee. All procedures performed in the study involving human participants were in accordance with the ethical standards of the institutional research committee and with the 1964 Helsinki declaration and its later amendments or comparable ethical standards.

\section{Scales}

Íkigai-9 Turkish Version Scale: This scale consists of nine items with a five-point Likert-type scale base ( $1=$ does not apply to me, $5=$ applies to me a lot). The scale the reasons of being through the dimensions of emotions toward life, attitudes toward the future, and the acknowledgement of one's own existence. The İkigai-9 was translated from Japanese and English into Turkish by four bilingual translators (Table 1).

The Warwick Edinburgh Mental Well-being Scale: This scale consists of seven items with a Likert-type five-point scale $(1=$ none of the time, $5=$ all of the time). The scale measures psychological functioning and emotional and mental well-being within the prior 2 weeks.

The Depression Anxiety Stress Scale (DASS-21): The DASS-21 Participants were asked about thier any recent feelings of depression, anxiety, and stress. Participants rate their experience of each along a four-point Likert-type scale $(0=$ "Never" and $3=$ "Almost always").

\section{Results}

According to the results of the Kolmogorov-Smirnov $(n>50)$ test, the total scores of scales and age are not distributed normally $(p<0.05)$. However, since the Skewness and Kurtosis values of these measurements show normal distribution ( \pm 1.5 range), it is appropriate to use Parametric tests in comparisons. Reliability analysis results of "scale questions" are given in Table 2 and Cronbach's Alpha value for the questions of all scales is over $75 \%$ shows that the reliability of these questions (items) is high.

The answers given to the Ikigai scale questions were subjected to Factor analysis. The reliability of the factor analysis was tested with the Kaiser-MeyerOlkin (KMO) test. The KMO coefficient was $88.7 \%$. Factor analysis test conducted according to the results of KMO test provides high reliability (0.887> 0.750). Bartlett test is also significant ( $<0.05)$. Looking at these two criteria, we can say that our data set is suitable for factor analysis. In the table 3 ; "Eigenvalues" statistics and "variance explanation powers of factors" are given.

In the study, the factors with significant initial eigenvalue statistics were determined. There is a 1-factor with eigenvalues greater than 1 . The first factor explains $49.8 \%$ of the total variance. According to the result of the factor analysis; the questions could be grouped "in a single dimension meaningfully in 1 factor". Comparison the results of all total scores of scales by gender were analysed and a statistically significant difference was found only in the comparison of total scores to the total scores of Anxiety Scale and Stress Scale $(p<0.05)$. In the table 4; "Correlation results of the scales" are given and correlation coefficients of the variables that are related to each other are shown with the asterisk (*) symbol.

A statistically significant relationship was observed among all scales and age $(p<0.05)$. In the table 5 ; Linear Regression analysis results are given to determine (predict) the effect of "Other Scales and Age" on "Ikigai score".

This analysis model was found significant $(\mathrm{p}<0.05)$ and the degree of accuracy was $54.8 \%$. When depression score increases an unit, the "Ikigai" score decreases by 0.156 points and this relationship was found statistically significant $(p<0.05)$. Similarly; when the mental well-being score increases by one unit, the ikigai score also increases by 0.775 points and this relationship has been found statistically significant $(p<0.05)$. Again, when the age decreases a unit, the "Ikigai" score decreases by 0.070 points and this relationship was found statistically significant $(p<0.05)$.

Despite that; when the anxiety score increases a unit, the ikigai score decreases by 0.008 points, but this relationship was not statistically significant ( $p>$ 0.05 ). Again, when the stress score increases a unit, the ikigai score decreases by 0.050 points, but this relationship was not statistically significant ( $p>$ 0.05).

\section{Discussion}

Apart from both İkigai-9 studies, including those in the UK and Japan, the present study found different correlations between anxiety, depression, stress, mental wellbeing, and ikigai in the Ikigai-9 Turkish version study. The findings from the present study could be different due to culture, ethnicity, or geograhic 
area differences, either totally, or to some extent. The more data collected from different studies and nations, the more we can understand the unknown aspects of human psychology and the related psyhological mechanisms. This will permit planning of efficient and effective, preferred therapies [5]. Posessing ikigai could be a protective factor against a number of negative health-related outcomes, including increased mortality rates and the presence of psychological burdens [15]. The positive side of Ikigai should be studied and affirmed in nations and areas other than the far East where its power and effective features have been noted in more detail. Thus, Ikigai could offer a solution for difficulties experienced by many people [16,17].

In the present study, we present the ikigai-9 Turkish version scale. This scale will be used as a tool for further studies, nationally and internationally. Similar to the UK study on the Ikigai- 9, the Turkish version did not support the three-dimensional structure originally proposed by Imai and colleagues in 2012 [10]. High Ikigai-9 Turkish version total scores were positively correlated mental well-being and negatively correlated with depression, anxiety, and stress; whereas for the Ikigai-9 UK total scores were not found to be correlated with any degree of anxiety or stress (Table 4).

These differing correlations imply that there are other factors present in the samples that attenuate the results. It is likely that these factors are related to cultures, religion, nationality, or other characteristics [7,17]. However, it has been found across cultures that the more ikigai one possesses, the more unlikely they are to commit suicide as outlined by one study; additionally; Japanese suicide prevention policies support any program aimed at enhancing a person's Ikigai in order to reduce suicide rates in Japan [18]. While the Linear Regression analysis revealed the effect of depression, mental well- being and age on ikigai as statistically significant, the stress and anxiety was not found as statistically significant in the same analysis (Table 5).

Ikigai is not only important in psychology; for example, within cardiovascular medicine a study utilizing a Japanese sample found that lkigai is associated with a lower risk of heart diseases $[4,5,19,20]$. People with higher levels of Ikigai experience lower cardiovascular problems in their lifetimes, which is an important fact as this disease type is a leading cause of mortality in almost every country.

Study Limitations: The present study has various limitations. First, we did not aquire information on the prevalence of mental illnesses such as anxiety or depression. Second, any disease or related medications or the lifestyle factors including nutrition, exercise or social interactions in which the participants engaged were not determined.

Prospects for further research: By this scale it could be possible to compare parameters related ikigai in other societies for a better understanding of geographical and cultural influence on well-being.

\section{Conclusions}

The İkigai-9 Turkish version scale has been validated for the Turkish population in this study. It was demonstrated that, as people age, their levels of İigai decrease; therefore, interventions that support and enhance İkigai should be encouraged by healthcare policies

\section{Declarations}

Acknowledgements

The authors would like to express deep gratitude to all co-workers for participating.

Declarations Conflict of Interest: The authors have no conflicts of interest to report.

Author Contributions: All authors contributed to the writing, reviewing and editing of the review.

Financial Disclosure: The authors declared that this study received no financial support.

\section{References}

1- Park, Y. Sense of "ikigai" (reason for living) and social support in the Asia-Pacific region.

Behaviormetrika, 2015;42(2):191-207. doi: 10.2333/bhmk.42.191.

2- $\quad$ Schippers MC, Ziegler N. Life Crafting as a Way to Find Purpose and Meaning in Life.

Front Psychol. 2019;10:2778. Published 2019 Dec 13. doi:10.3389/fpsyg.2019.02778

3- $\quad$ Mori, K., Kaiho, Y., Tomata, Y., Narita, M., Tanji, F., Sugiyama, K., Sugawara, Y., et al. Sense of life worth living (ikigai) and incident functional disability in elderly Japanese: The Tsurugaya Project. Journal of Psychosomatic Research, 2017;95:62-67. doi: 10.1016/j.jpsychores.2017.02.013.

4- $\quad$ Megumi, K., Ito, H., Kaneko, Y., \& Motohashi, Y. Effect of having a sense of purpose in life on the risk of death from cardiovascular diseases. Journal of Epidemiology, 2008;18(5):191-196. doi: 10.2188/jea.je2007388 
5- $\quad$ Tanno, K., Sakata, K., Ohsawa, M., Onoda, T., Itai, K., Yaegashi, Y., JACC Study Group., Associations of İigai as a positive psychological factor with all-cause mortality and cause- specific mortality among middle-aged and elderly Japanese people: findings from the Japan Collaborative Cohort Study. Journal of Psychosomatic Research, 2009;67(1), 67-75. doi: 10.1016/j.jpsychores.2008.10.018.

6- Ishida R., Reducing anxiety in stutterers through the association between "purpose in life/ikigai" and emotions. Global Journal of Health Science, 2012;4(5):120-124. doi: 10.5539/gjhs.v4n5p120

7- $\quad$ Shirai, K., Iso, H., Fukuda, H., Toyoda, Y., Takatorige, T., \& Tatara, K. (2006). Factors associated with "ikigai" among members of a public temporary employment agency for seniors (Silver Human Resources Centre) in Japan; Gender differences. Health \& Quality of Life Outcomes, 4(12). doi: $10.1186 / 1477-7525-4-12$

8- $\quad$ Fuster, J.M., The prefrontal cortex and its relation to behavior. Progress in Brain Research, 1991;87:201-211. doi: 10.1016/s0079-6123(08)63053-8

9- Ingvar, D.H., "Memory of the future": An essay on the temporal organization of conscious awareness. Human Neurobiology, 1995;4(3):127-136. PMID: 3905726

10- Imai, T., Osada, H., Nishi, S., The reliability and validity of a new scale for measuring the concept of ikigai (ikigai-9). Japanese Journal of Public Health, 2012; 59(7): 433-439.

PMID: 22991767

11- Fido, D., Kotera, Y., \& Asano, K. English translation and validation of the İkigai-9 in a UK sample. International Journal of Mental Health and Addiction, 2019, doi: 10.1007/s11469-019-00150-w

12- Demirtas, S., Baytemir, K. Adaptation of Warwıck-Edınburgh Mental Well-Beıng Scale short form into Turkısh: Validity and reliability study. Electronic Journal of Social Sciences, 2019;18(70):664-666. Retrieved 13 April 2020 https://dergipark.org.tr/tr/download/article- file/672580

13- Yılmaz, Ö., Boz, H., Arslan, A ., DASS 21 Turkish version Scale A validity and reliability study. Finans Ekonomi ve Sosyal Araştırmalar Dergisi (FESA), 2017;2(2):78-91. Retrieved 13 April 2020 from https://dergipark.org.tr/tr/pub/fesa/issue/30912/323190

14- Faul, F., Erdfelder, E., Lang, A.G., \& Buchner, A. G*Power 3: a flexible statistical power analysis program for the social, behavioral, and biomedical sciences. Behavioral Research Methods, 2007;39(2):175-191. doi: 10.3758/BF03193146

15- Hasegawa, A., Fujiwara, Y., Hoshi, T., Shinkai, S, Regional differences in İkigai (reason(s) for living) in elderly people - relationship between İkigai and family structure, physiological situation and functional capacity. Nihon Ronen Igakkai Zasshi, 2003;40(4):390-396.

16- Alimujiang, A., Wiensch, A., Boss, J., Fleischer, N.L., Mondul, A.M., McLean, K., et al. Association between life purpose and mortality among US adults older than 50 years. JAMA Netw Open, 2019;2(5):e194270. doi:10.1001/jamanetworkopen.2019.4270 
18- Saint Arnault, D., Shimabukuro, S. Floating on air: Fulfillment and self-in-context for distressed Japanese women. Western Journal of Nursing Research, 2016; 38(5):572-595. doi: 10.1177/0193945915625219

19- Musich, S., Wang, S. S., Kraemer, S., Hawkins, K., Wicker, E., Purpose in life and positive health outcomes among older adults. Population Health Management,

2019; 21(2):139-147. doi: 10.1089/pop.2017.0063

20- Sone, T., Nakaya, N., Ohmori, K., Shimazu, T., Higashiguchi, M., Kakizaki, M., et al. Sense of life worth living (ikigai) and mortality in Japan: Ohsaki study. Psychosomatic Medicine, 2008;70:709-715. doi: 10.1097/PSY.0b013e31817e7e64.

\section{Tables}

Tables 1-5 are in the supplementary files section.

\section{Supplementary Files}

This is a list of supplementary files associated with this preprint. Click to download.

- Tables.docx 